\title{
La investigación sobre trayectorias educativas
}

\section{Research on educational trajectories}

DOI: $10.46932 / \mathrm{sfjdv2n1-005}$

Received in: November 1st, 2020

Accepted in: December 30th, 2020

\author{
Abraham Bernárdez-Gómez \\ Investigador Predoctoral (REF: BES-2017-081040) \\ Institución: Universidad de Murcia \\ Dirección: C/ Campus Universitario, 12, 30120. Facultad de Educación \\ E-mail: abraham.bernardez@um.es
}

\section{RESUMEN}

El objetivo desarrollado en esta investigación ha sido: explorar y describir la investigación anterior que guarde relación con el estudio de las trayectorias educativas. Ello es debido a que el presente texto se enmarca dentro de la tesis doctoral del autor, sobre trayectorias educativas de estudiantes en riesgo de exclusión, siendo un paso necesario en la misma, una mirada atrás, hacía las investigaciones semejantes para continuar con la tradición anterior. Para realizar la tarea planteada se ha propuesto, a modo de metodología, el desarrollo de una revisión sistemática de literatura en las principales bases de datos del ámbito educativo. Una vez rescatadas las diferentes referencias, se ha procedido a realizar un análisis de contenido de las mismas, en base a cuatro categorías: temas de investigación, metodología empleada, técnicas de recogida de datos empleadas y características de las muestras seleccionadas. Una vez realizado el análisis y la discusión de los datos, se han llegado a diferentes conclusiones, entre ellas: los estudiantes en riesgo de exclusión educativa son el foco principal para el estudio de las trayectorias; la manera de abordar dichas investigaciones es, fundamentalmente, mediante un enfoque biográfico-narrativo; la terminología existente en este ámbito es diversa.

Palabras clave: Teoría del curso de vida, trayectoria educativa, historiad de vida, jóvenes en riesgo, metodología cualitativa.

\begin{abstract}
The objective developed in this research has been: to explore and describe the previous research related to the study of educational trajectories. This is because this text is part of the author's doctoral thesis, on educational trajectories of students at risk of exclusion, being a necessary step in it, a look back, doing similar research to continue the previous tradition. In order to carry out the task, it has been proposed, as a methodology, to develop a systematic review of literature in the main databases of the educational field. Once the different references have been rescued, a content analysis of the references has been carried out, based on four categories: research topics, methodology used, data collection techniques used and characteristics of the selected samples. Once the analysis and discussion of the data has been carried out, different conclusions have been reached, including: students at risk of exclusion of education are the main focus for the study of trajectories; the way to approach such research is primarily through a biographicalnarrative approach; the terminology in this area is diverse.
\end{abstract}

Keywords: Life course theory, educational trajectory, life history, young people at risk, qualitative methodology. 


\section{INTRODUCCIÓN}

Una cuestión ineludible en todo proceso de investigación es volver la mirada atrás para escudriñar investigaciones similares a las que se comienza. Son referentes que permitirán, situar el propio estudio en torno a los ejes teóricos que se deben considerar para su desarrollo, así como orientar cómo plantear el diseño metodológico más adecuado para llevar a cabo el proceso de investigación. Por ello, el presente artículo se sitúa dentro del contexto de una tesis doctoral dónde se estudiarán las trayectorias educativas de alumnado vulnerable. De esta forma, el objetivo que se ha planteado llevar a cabo este trabajo ha sido: explorar y describir la investigación anterior que guarde relación con el estudio de las trayectorias educativas. La principal finalidad que se propone para este estudio no es otra que averiguar cuál es la costumbre en el estudio de esta problemática educativa. Además de las trayectorias en si mismo, se ha apuntado como tema central de la tesis doctoral al alumnado vulnerable. Esto se debe a que el ámbito de estudio presente en el artículo se relaciona de manera recurrente con otros problemas de relevancia en el ámbito escolar, como son el alumnado en riesgo de exclusión, la falta de implicación de los estudiantes, el absentismo, abandono escolar o fracaso de la escuela, entre otros.

El estudio de las trayectorias educativas tiene su base, o al menos así se considera por nuestra parte, en la Teoría del Curso de Vida (LCT, por sus siglas en inglés), constructo que ha ido ampliamente estudiado, tanto en el ámbito de las ciencias sociales (Duque y Silva 2013; Mortimer y Shanahan, 2003; Escobedo, Fusulier, y Tremblay, 2017 Gebel y Heineck, 2019), como en el ámbito educativo (Blasbichler y Vogt, 2020; Christodoulou, Bousia y Kiprianos, 2018; Portela, Nieto y Torres, 2019). Este curso de vida, en palabras de Crosnoe y Benner (2016), consiste en el "pasaje de una persona a través de una secuencia de roles calificados por edad y contextos con tareas asociadas a su desarrollo" (p. 180) describiendo, con el paso del tiempo, los diferentes roles de la persona, hechos que tienen lugar, las transiciones que atraviesa, la trayectoria que desarrolla.

Siendo unos de los investigadores de referencia en el ámbito, aunque no los únicos, Hutchison (2019) o Mortimer y Shanahan (2003) describen los principales componentes de la LCT de forma precisa: el colectivo o cohorte al que pertenece la muestra objeto de estudio. Puesto que, se entiende, la existencia de un grupo que experimenta hechos similares al mismo tiempo en el curso de su vida; los eventos de $v i d a$, diferentes hechos que ocurren que pueden significar pequeñas adaptaciones en la trayectoria de una persona; los eventos críticos o puntos de inflexión, denominándose así los diferentes hechos que revisten la gravedad suficiente para alterar de forma sustancial la trayectoria del individuo, provoca una desestabilización que lleva a que se produzca el siguiente elemento en la LCT; las transiciones, se entienden como procesos de cambio en el estado del sujeto e incluso en su identidad, el tiempo que transcurre entre ellas es directamente proporcional a la estabilidad del sujeto, además marca el curso de; 
las trayectorias, la secuencia de roles y eventos en la vida del sujeto, son múltiples en función del ámbito dónde se desarrollan, en el caso que nos interesa, las educativas.

Así pues, en el trabajo que aquí se desarrolla, se pondrá en el centro de la cuestión cómo se han desarrollado las investigaciones en torno a las trayectorias educativas de los estudiantes en riesgo, los que han sido abandonados por el sistema y, así se podrá observar en el análisis de datos, muestran su historia después de pasar por diferentes situaciones indeseables.

\section{METODOLOGÍA}

Con el fin de alcanzar el objetivo planteado para esta investigación: explorar y describir la investigación anterior que guarde relación con el estudio de las trayectorias educativas. Se ha realizado una revisión sistemática de literatura (Pacios, 2013), una investigación perteneciente al paradigma cualitativo (Tejedor, 2020; Triana y Parra, 2020; Trillo, Parada y Bernárdez-Gómez, 2020), considerada de naturaleza secundario por la obtención de sus datos a partir de investigaciones ya realizadas que son analizadas, por lo general, mediante la técnica de análisis de contenido (Chasco, 2020; Valladolid e Chávez, 2020). De manera particular, el proceso de investigación llevado a cabo con el enfoque de revisión tiene unas características específicas: establecer los criterios que serán usados en la búsqueda, recoger y realizar un análisis preliminar de los documentos para seleccionar los usados en la investigación y, para finalizar realizar el análisis final de los textos (Bernárdez-Gómez, Marafante y Da Silva, 2020, p.75650).

\subsection{MUESTRA}

Para abordar este artículo se ha realizado una búsqueda de textos académicos en los que se da cuenta de investigaciones sobre las trayectorias en el ámbito educativo. Debiendo apuntar que tal búsqueda no ha sido sencilla debido a que en las bases de datos consultadas emergían pocas investigaciones realizadas con cierta actualidad. Una falta de documentación para analizar que ha llevado a la inferencia de la escaseza de investigación en este ámbito. Aunque no se hará una especulación de la causa subyancente, bien podría ser porque es un problema educativo que no interesa, o bien ya se encuentra ampliamente estudiado.

Sin entrar en mayores disquisiciones, para abordar la investigación se han utilizado las bases de datos de referencia en el ámbito educativo: Dialnet, Web of Sciencie y Linceo+UNED. Al acceder a ellos se han usado los descriptores establecidos para la búsqueda: \#trayectoria educativa, \#trayectoria escolar, \#historias de vida. Igualmente, se han aplicado unos filtros de búsqueda básicos con el fin de facilitar la selección de las referencias, estas fueron: textos en acceso abierto, redactados en español o inglés, 
publicados en los últimos 5 años, referencias del ámbito educativo. Una vez realizada la primera búsqueda se seleccionaron 8 referencias para realizar el análisis.

A continuación se puede encontrar la tabla 1, dónde se esquematizan los resultados de la búsqueda mencionando a los autores, los descriptores -que son muestra de las temáticas estudiadas-, la metodología empleada en la investigación, así como las técnicas de recogida de datos y el tipo de muestra escogido para ello. Estos aspectos son los que servirán de guía para estructurar el análisis posterior, esto es, las categorías de análisis que se han establecido.

Tabla 1: Referencias extraídas para el análisis.

Fuente: elaboración propia.

\subsection{PROCESO DE ANÁLISIS DE DATOS}

\begin{tabular}{|c|c|c|c|}
\hline REFERENCIA & DESCRIPTORES & METODOLOGÍA & $\begin{array}{l}\text { TÉCNICAS DE } \\
\text { RECOGIDA DE } \\
\text { DATOS }\end{array}$ \\
\hline $\begin{array}{l}\text { De la Cruz y } \\
\text { Ilinich (2019) }\end{array}$ & $\begin{array}{l}\text { Abandono escolar, Educación media } \\
\text { Superior, Exclusión educativa, } \\
\text { Experiencias de los estudiantes, } \\
\text { Retorno escolar, Trayectoria escolar }\end{array}$ & Cualitativa & Grupo de discusión \\
\hline $\begin{array}{c}\text { Emery, Spruyt, } \\
\text { Boone y Van } \\
\text { Avermaet } \\
(2020)\end{array}$ & $\begin{array}{l}\text { Equidad; estudiantes migrantes; } \\
\text { capital cultural; sentido de } \\
\text { derecho; Estratégica } \\
\text { conocimiento; Logro }\end{array}$ & $\begin{array}{c}\text { Cualitativa } \\
\text { Biográfico/narrativo }\end{array}$ & Entrevista en profundidad \\
\hline Feito (2015) & $\begin{array}{l}\text { Abandono escolar, educación de } \\
\text { adultos, educación y empleo, } \\
\text { etnografía } \\
\text { educativa, reforma educativa }\end{array}$ & $\begin{array}{c}\text { Cualitativa } \\
\text { Historias de vida }\end{array}$ & Entrevista \\
\hline $\begin{array}{l}\text { Montes Ruiz } \\
\quad \text { (2016) }\end{array}$ & $\begin{array}{l}\text { Reenganche educativo; Abandono } \\
\text { escolar prematuro; Retorno } \\
\text { educativo; Desapego escolar; } \\
\text { Adhesión educativa. }\end{array}$ & $\begin{array}{c}\text { Cualitativa } \\
\text { Biográfico/narrativo }\end{array}$ & $\begin{array}{c}\text { Entrevista } \\
\text { semiestructurada }\end{array}$ \\
\hline $\begin{array}{l}\text { Morentin y } \\
\text { Ballesteros } \\
\quad(2018)\end{array}$ & $\begin{array}{l}\text { Deserción escolar; Necesidades } \\
\text { educacionales; Educación no formal; } \\
\text { Autoaprendizaje; Escuela. }\end{array}$ & $\begin{array}{c}\text { Cualitativa } \\
\text { Historias de vida }\end{array}$ & $\begin{array}{c}\text { Entrevista } \\
\text { semiestructurada }\end{array}$ \\
\hline $\begin{array}{l}\text { Morentin y } \\
\text { Ballesteros } \\
\quad(2019)\end{array}$ & $\begin{array}{l}\text { Abandono temprano de la educación } \\
\text { y formación, igualdad de } \\
\text { oportunidades educativas, política } \\
\text { educativa, narrativas. }\end{array}$ & $\begin{array}{c}\text { Cualitativa } \\
\text { Biográfico/narrativo }\end{array}$ & $\begin{array}{c}\text { Entrevista } \\
\text { Grupo focal } \\
\text { Historia de vida }\end{array}$ \\
\hline $\begin{array}{l}\text { Nieto, Portela, } \\
\text { Torres y } \\
\text { Rodríguez } \\
(2018)\end{array}$ & $\begin{array}{l}\text { Abandono educativo, Fracaso escolar, } \\
\text { Jóvenes desfavorecidos, Estudiantes } \\
\text { en Riesgo, Renganche formativo, } \\
\text { Análisis de Trayectoria, Historias de } \\
\text { vida. }\end{array}$ & $\begin{array}{c}\text { Cualitativa } \\
\text { Estudio de caso }\end{array}$ & $\begin{array}{c}\text { Entrevista en profundidad } \\
\text { semiestructurada } \\
\text { Biogramas }\end{array}$ \\
\hline $\begin{array}{l}\text { Thiele et al. } \\
\text { (2017) }\end{array}$ & $\begin{array}{l}\text { Desventaja; compromiso; identidad; } \\
\text { educación superior }\end{array}$ & Cualitativa & $\begin{array}{c}\text { Entrevista en profundidad } \\
\text { semiestructurada }\end{array}$ \\
\hline
\end{tabular}

En investigaciones anteriores dónde se ha utilizado una metodología similar (Alarcón et al., 2017; Bernárdez-Gómez y Belmonte, 2020) se ha realizado un proceso de reducción y análisis de datos 
siguiendo la técnica de análisis de contenido, además de realizar una codificación de carácter inductivodeductivo, aunque se poseían unas categorías preestablecidas no se ha cerrado la puerta a la codificación con otras diferentes. Además, con la ayuda el programa de análisis cualitativo ATLAS.ti V9 se ha realizado una codificación en tres etapas: abierta, axial y selectiva. Finalmente, se ha realizado un análisis de datos en base a las siguientes categorías: temas de investigación, metodología empleada, técnicas de recogida de datos empleadas y características de las muestras seleccionadas.

\section{ANÁLISIS DE RESULTADOS Y DISCUSIÓN}

\subsection{TEMAS DE INVESTIGACIÓN}

En el desarrollo de las investigaciones que aquí se han analizado existe un problema de estudio que ha sido recurrente en la mayoría de ellas y que ejerce una función de eje vertebral en las trayectorias educativas, el abandono escolar.

Como se puede constatar, los estudios realizados por De la Cruz y Ilinich (2019), Feito (2015), Montes Ruiz (2016), y Nieto, Portela, Torres y Rodríguez (2018), basan la investigación en la trayectoria educativa que realiza el alumnado que ha vuelto a sus estudios después de un episodio de abandono escolar. Estos trabajos se sustentan en el análisis de las experiencias de los estudiantes a partir de la recuperación de sus vivencias, sentidos y significados en torno al abandono y retorno escolar, analizando elementos relacionados con el abandono escolar, procesos acaecidos al estudiante mientras estuvo al margen del sistema escolar, las razones por las que regresó y su percepción del contexto escolar, de la educación y de sí mismo, después de regresar a la institución escolar. Estos elementos permiten elaborar una línea temporal para una mejor visualización de la trayectoria escolar del alumno, lo que, por otro lado, hace viable una reconstrucción de los momentos clave que desencadenaron tanto el abandono escolar, como el retorno.

Con ello, se pretende averiguar las causas que condujeron al alumnado a situaciones de abandono o fracaso escolar, investigando las diversas trayectorias hacia el abandono escolar de personas que dejaron sus estudios en la educación obligatoria, con o sin, alcanzar el titulo equivalente a la Educación Secundaria Obligatoria. Concretamente, se examina el caso de individuos que, tras varios años de experiencia fuera de los estudios, deciden acudir de nuevo a instituciones educativas, bien en los Centros de Educación de Personas Adultas, donde pueden proseguir el itinerario formativo ordinario, o en medidas consideradas de segunda oportunidad.

Se quiere destacar las investigaciones realizadas por Morentin y Ballesteros (2018, 2019), las cuales presentan una singularidad con respecto a las otras, aun poniendo el foco en el mismo problema educativo. Los dos textos de estos autores tratan de comprender el proceso de abandono a través de 
trayectorias de vida, al igual que los anteriores. Sin embargo, también tratan de valorar los diferentes aprendizajes adquiridos para inferir estrategias educativas que puedan ser aplicadas en entornos escolares, logrando motivación, sentimiento de pertenencia, atención, etc. y con ello, contribuir a la igualdad de oportunidades y equidad educativa.

Con temas relacionados como la equidad o alumnos en situación de riesgo, entre otros, pero en una dirección distinta a las anteriores, se presentan los trabajos de Emery, Spruyt, Boone y Van Avermaet (2020) y Thiele et al. (2017). El primero de ellos se centra en evaluar en qué medida son relevantes dos elementos del capital cultural (sentido de derecho y conocimiento estratégico), al estudiar las trayectorias educativas de los estudiantes migrantes recién llegados. Y, por su parte, el trabajo de Thiele et al. (2017), ahonda sobre el por qué algunas personas de entornos desfavorecidos tienen éxito a la hora de ser admitidas en prestigiosas instituciones de educación superior. Por ello se puede señalar que la totalidad de los trabajos hacen referencia a una problemática común, el estudio de las trayectorias del alumnado que se encuentra en desventaja con respecto al sistema educativo.

\subsection{METODOLOGÍA EMPLEADA}

Profundizando en los aspectos que hacen referencia al diseño, aunque haya pequeñas diferencias en cuanto a la explicitación o concreción de la metodología empleada en la revisión que se está realizando se puede decir que hay un pensamiento mayoritario, las trayectorias de los estudiantes se estudian a través de la narrativa, del discurso de los individuos. Dichas divergencias están referidas, fundamentalmente, a la concreción dentro del enfoque cualitativo. Así, algunos investigadores (De la Cruz y Ilinich, 2019; Thiele et al., 2017) indican únicamente que el método es cualitativo y otros profundizan más dentro de los enfoques que podemos encontrar dentro de lo cualitativo haciendo referencia a la investigación biográfico-narrativa o a las historias de vida. Una cuestión reforzada con la finalidad que estos investigadores persiguen, "comprender las diferentes complejidades y realidades de personas etiquetadas" [...] siendo "la metodología cualitativa la más adecuada para adentrarse en el mapa de significados desde el cual los sujetos entienden y perciben el mundo" (Morentin y Ballesteros, 2018, p. 9). La idea fundamental es reconstruir las distintas percepciones y valoraciones personales y sociales desde la mirada directa del informante. Con ello se explora la respuesta y percepción del individuo y la relación que ha mantenido el sujeto con la sociedad ante las dinámicas de cambio y en su trayectoria (Montes Ruiz, 2016).

Para ilustrar lo anterior, hay autores que basan la investigación en una serie de preguntas que sirven para indagar en los detonantes del alejamiento de sus estudios. Es el caso de De la Cruz y Ilinich (2019), autores que han desarrollado su trabajo mediante un análisis cualitativo orientado por la tradición fenomenológica y centrado en el análisis de las experiencias de los sujetos, los cuales parten de las 
siguientes cuestiones de investigación: ¿por qué abandonaron sus estudios?, ¿qué hicieron mientras estuvieron fuera del contexto escolar?, ¿por qué decidieron volver a estudiar?, ¿cómo viven ahora la educación que les ofrece la institución?

Igualmente Feito (2015) y Montes Ruiz (2016) diseñaron la investigación en torno a una serie de ejes temáticos, los cuales reconstruían historias de vida y así las trayectorias educativas y profesionales de cada entrevistado: ¿qué factores generan un cambio en las trayectorias educativas hacia el desenganche y el reenganche educativo?, ¿qué razones le impulsaron a volver a la escuela y al centro en concreto en el que estaba matriculado?, ¿qué dificultades ha sufrido en cuanto al aprendizaje, relación con los compañeros y el profesorado, actividades culturales que realizan, etc.?, ¿qué condiciona esas decisiones y cómo afectan estas al desarrollo de su trayectoria social y personal? Todo ello con el fin de dilucidar elementos generadores de cambio en las trayectorias educativas hacia el reenganche, así como identificar diversas motivaciones que provocaron el desenganche educativo previo.

En suma, lo pretendido mediante la elección de este tipo de metodología es adentrarse en las percepciones sobre el papel de la educación en la vida de los jóvenes (Morentin y Ballesteros, 2018) y así reconstruir historias personales de los sujetos y desarrollar una comprensión de la experiencia vivida (Thiele et al., 2017) reconstruyendo narrativamente sus trayectorias educativas (Emery, Spruyt, Boone y Van Avermaet, 2020).

\subsection{TÉCNICAS DE RECOGIDA DE DATOS.}

En cuanto a las técnicas de recogida de información se refiere también existe coincidencia por parte de los autores de los textos analizados. Todos ellos han empleado la técnica de la entrevista a excepción de De la Cruz y Ilinich (2019) que, para recabar la información emplearon la técnica de grupos focales, de una hora y media de duración.

Con respecto a las entrevistas utilizadas por el resto de investigadores, existe una pequeña diferencia entre el tipo de entrevista utilizada. Los autores analizados utilizaron la técnica de-entrevista semiestructurada y otros estructurada. Igualmente, algunos autores formularon varias entrevistas a un mismo sujeto, obteniendo un feedback por parte del investigador que permitió una reflexión conjunta con los dos protagonistas de la entrevista. Así mismo, todas las entrevistas se consideraban en profundidad.

De esta forma, para las semiestructuradas utilizaban un listado de temas (Emery, Spruyt, Boone y Van Avermaet, 2020) que se convertían en un guion previo a modo de orientación (Morentin y Ballesteros, 2018), y para las estructuradas preguntas cerradas (Feito, 2015). Todas ellas basadas en preguntas que generaron historias sobre las experiencias, antes y después de retomar su educación para alumnado reincorporado, junto con las decisiones y acciones que los alumnos tomaron durante su trayectoria 
educativa. Esto permite la reconstrucción secuencial de la historia que queda a merced del entrevistado siendo el investigador quien orienta, mientras que el entrevistador se posiciona en un segundo plano, reconstruyendo y otorgando significado secuencial a la narración principal (Montes Ruiz, 2016).

A través de lo explorado a través de las lecturas, se puede extraer que se aportara un resultado enriquecedor y que facilitaba una mayor interacción con la persona cuya trayectoria se investigaba era la entrevista semiestructurada de carácter cíclico, esto es, que se repetían. Señalando Morentin y Ballesteros que "una buena entrevista es aquella en la que se obtienen buenas preguntas a partir de las respuestas compartidas" (2018, p. 10) por lo que se hace necesario realizar, al menos, dos entrevistas para desarrollar las historias de vida.

En otra dirección, Montes Ruiz (2016), Nieto, Portela, Torres y Rodríguez (2018) y Morentin y Ballesteros (2019) emplearon diferentes técnicas con las que complementar las entrevistas. Por su parte, Montes Ruiz (2016) y Nieto, Portela, Torres y Rodríguez (2018) emplearon el biograma o life-grid, la cual ayuda a la reconstrucción de los diferentes hechos, generando una estructura de ordenación de la información, facilitando "la construcción de fechas y eventos desconocidos en relación a aquellos eventos conocidos mejorando sustancialmente la coherencia, la precisión y la exactitud” (Montes Ruiz, 2016, p. 12). Una técnica destacada por el autor como un buen complemento para la entrevista debido a las características mencionadas. Y, al igual que De la Cruz y Ilinich (2019), Morentin y Ballesteros (2019) emplearon los grupos de discusión.

\subsection{CARACTERÍSTICAS DE LA MUESTRA}

Para finalizar este capítulo se hará referencia a las distintas muestras empleadas en el desarrollo de las investigaciones que se están analizando. Como en puntos anteriores, también existe coincidencia a la hora de seleccionar los sujetos que formarán parte de la muestra. El proceso de elección se ha realizado mediante un tipo de muestreo no probabilístico deliberado, también conocido como, muestreo por conveniencia o posibilidad de acceso.

Pese a la coincidencia en el método de selección de la muestra, cada uno de los autores ha utilizado diferentes criterios para los alumnos en lo que se refiere a edad, sexo, estudios que cursaban en la actualidad, u otras características sociodemográficas que eran relevantes respectivamente para cada uno de los estudios. Igualmente, el número total de sujetos que componía la muestra también difería entre cada una de las investigaciones, desde los seis sujetos encontrados en la investigación con menor muestra (Thiele et al., 2017), hasta los cien participantes de la que contaba con mayor número (Feito, 2015). Sin embargo, en todos ellos podemos encontrar, al menos, dos coincidencias. 
La primera haría referencia a que todos los sujetos escogidos se encuentran dentro de lo que se denominan sujetos en riesgo (Nieto, Portela, Torres y Rodríguez, 2018). Esos alumnos que por alguna particularidad que presentan, bien sea a nivel socioeconómico, familiar o contextual, pueden ser más vulnerables que otros y así, desarrollarse situaciones indeseadas, como desenganche, abandono o fracaso escolar.

La segunda observa el tipo de centro en el que se encuentran los alumnos, puesto que no se trata de contextos en los que se desarrolla una trayectoria lineal, al ser centros donde se desarrollan medidas de segunda oportunidad -dígase centros con FPB, AO, PFP- (Nieto, Portela, Torres y Rodríguez , 2018; Thiele et al., 2017) o centros que facilitan la continuación con sus estudios -ESA, educación para adultos(Emery, Spruyt, Boone y Van Avermaet, 2020; Feito, 2015).

\section{CONCLUSIONES}

Los diferentes estudios de carácter biográfico, o que se pueden enmarcar dentro del enfoque biográfico-narrativo han supuesto una bandera importante dentro las investigaciones de carácter cualitativo. Las cuales, con la aparición de paradigmas centrados en las personas y en el desarrollo de su vida como son la Teoría del Curso de Vida y las trayectorias que encontramos en ella, han visto reforzados sus planteamientos y su forma de hacer investigación y generar conocimiento.

Como se ha visto a lo largo del texto, aunque la investigación sea escasa, seguramente porque es un campo en el que abrir caminos, hay una serie de cuestiones que tienen en común todas las investigaciones estudiadas:

- El tema principal de estudio está íntimamente relacionado con la cohorte en al que se pone el foco, el alumno en riesgo de exclusión educativa que ha pasado por dificultades en su trayectoria, bien por haberse alejado de su formación de una manera o de otra y que después, en la mayoría de las veces, se ha vuelto a incorporar a ella.

- La forma de abordar dichas investigaciones, de la que ya se ha señalado que es mediante un paradigma cualitativo y, de manera concreta, un enfoque biográfico-narrativo. Sin embargo, la elección dominante de los investigadores se centra en llevarlas a cabo mediante historias de vida, como forma de recuperar la biografía de los estudiantes y, en ella, identificar los distintos eventos de vida y eventos críticos que han generado transiciones, alterando, de esta forma, la trayectoria escolar de los estudiantes.

- Pese a que las coincidencias en base a términos y conceptos encontradas en los textos sean superiores a las diferencias, se han encontrado ciertas discrepancias si nos referiremos a este aspecto a este aspecto. 
En último término, solo resta apuntar que se trata de un campo de investigación centrado en la persona y en su vivencia directa, por lo que aporta una gran riqueza de producto de investigación. Siendo, algunas de sus posibles líneas de investigación, de interés para su indagación: como las posibles relaciones entre los eventos críticos y de vida y como estos se desarrollan en las transiciones; la existencia o no de factores que puedan minorar el impacto de los eventos mencionados; o la correlación entre eventos de diferentes escenarios y su impacto en las diferentes trayectorias que desarrolla el individuo. 


\section{REFERENCIAS}

Alarcón, A., Munera, L. y Montes, A. (2017). La teoría fundamentada en el marco de la investigación educativa. Saber, ciencia y libertad, 12(1), 236-245. https://doi.org/10.18041/23823240/saber.2017v12n1.1475

Bernárdez-Gómez, A. y Belmonte, M. L. (2020). Vulnerabilidad y exclusión educativa, una problemática postergada. Revista Interdisciplinar em Gestão, Educação, Tecnologia e Saúde-GETS, 3 (1), 79-94.

Bernárdez-Gómez, A., Marafante, G. y Da Silva, L. (2020). Perspectivas teóricas sobre o engajamento agêntico. Braz. J. of Develop., Curitiba, 6(10), 75648-75661. https://doi.org/10.34117/bjdv6n10-116

Blasbichler, A. A., y Vogt, M. (2020). Between and beyond. The course of a life in the realms of history of education, general pedagogy and comparative studies. Interview with Edwin Keiner. Espacio, Tiempo y Educación, 7(2), 235-247. https://doi.org/10.14516/ete.380

Chasco, S. R. (2020). El rigor de lo cualitativo: Las obligaciones empíricas de la interpretación socioantropológica. REIS: Revista Española de Investigaciones Sociológicas, 170, 167-171.

Christodoulou, M., Bousia, C., y Kiprianos, P. (2018). The life-course formation of teachers' profession. How emotions affect VET teachers' social identity. Revista de Sociología de La Educación-RASE, 11(2), 280-296. https://doi.org/10.7203/rase.11.2.12300

Crosnoe, R., y Benner, A. D. (2016). Educational Pathways. En M. J. Shanahan, J. T. Mortimer, y M. Kirkpatrick Johnson (Eds.), Handbook of the Life Course: Volume II (pp. 179-200). Springer International Publishing. https://doi.org/10.1007/978-3-319-20880-0_8

De La Cruz, G. Y Ilinich, D. (2019). “¿Por qué regresé a la escuela?” Abandono y retorno escolar desde la experiencia de jóvenes de educación media superior. Perfiles Educativos, 51(165), 8-26. https://doi.org/10.22201/iisue.24486167e.2019.165.58713

Duque, A. Do N. y Silva, I. R. Da. (2013). Papeis sociais e envelhecimento em uma perspectiva de curso de vida. EDUCAmazônia, 11(1), 310-326, https://doi.org/10.1590/s0102-37722000000100005

Emery, L., Spruyt, B., Boone, S. Y Van Avermaet, P. (2020). The educational trajectory of Newly Arrived Migrant Students: Insights from a structural analysis of cultural capital. Educational Research, 62(1), 1834. https://doi.org/10.1080/00131881.2020.1713021

Escobedo, A., Fusulier, B. y Tremblay, D. G. (2017). Work and family articulation: A life course approach in different professional and institutional contexts. VII Congrés Català de Sociologia i V Congrés Català de Joves Sociòlegs / Sociòlogues: Llibre de Resums de Les Sessions Dels Grups de Treball, 2017, 93. https://dialnet.unirioja.es/servlet/articulo?codigo $=6515861$

Feito, R. (2015). La ESO de adultos. Trayectorias de abandono escolar temprano entre estudiantes con experiencia laboral. Profesorado. Revista de curriculum y formación del profesorado, 19(2), 351-371.

Gebel, M. y Heineck, G. (2019). Returns to education in the life course. Research Handbook on the Sociology of Education. https://www.elgaronline.com/view/edcoll/9781788110419/9781788110419.00035.xml 
Hutchison, E. D. (2019). An Update on the Relevance of the Life Course Perspective for Social Work. Families in Society, 100(4), 351-366. https://doi.org/10.1177/1044389419873240

Montes Ruiz, A. (2016). En la encrucijada. Factores de desenganche y reenganche educativo en la trayectoria de seis jóvenes en Cataluña (España). INGURUAK, 61, 1-22.

Morentin, J. Y Ballesteros, B. (2019). ¿Igualdad de oportunidades? Más que el mero acceso: narrativas de jóvenes con trayectorias de fracaso y abandono temprano de la educación en España y Portugal. Revista Fuentes, 21(2), 143-159. https://doi.org/10.12795/revistafuentes.2019.v21.i2.01

Morentin, J. Y Ballesteros, B. (2018). Desde Fuera de la Escuela: Una Reflexión en torno al Aprendizaje a partir de Trayectorias de Abandono Escolar Prematuro. REICE. Revista Iberoamericana sobre Calidad, Eficacia y Cambio en Educación, 16(1), 5-20. https://doi.org/10.15366/reice2018.16.1.001

Mortimer, J. T., y Shanahan, M. J. (Eds.). (2003). Handbook of the Life Course. Springer US. https://doi.org/10.1007/b100507

Nieto, J. M., Portela, A., Torres, A. y Rodríguez, M. J. (2018). Del abandono educativo temprano al reenganche formativo: un estudio narrativo con biogramas. Educatio Siglo XXI, 36(2), 93-114. https://doi.org/10.6018/j/333041

Pacios, A. (2013). Técnicas de búsqueda y uso de la información. Editorial Universitaria Ramón Areces. Portela Pruaño, A., Nieto Cano, J. M. y Torres Soto, A. (2019). La reincorporación formativa de jóvenes que abandonan tempranamente la educación: relevancia de su trayectoria previa. Revista Española de Pedagogía, 77 (272), 103-121. https://doi.org/10.22550/REP77-1-2019-07

Tejedor, J. A. H. (2020). Knowing Oldest Old's Preferences May Improve their Healthcare: A Qualitative Study. Revista Iberoamericana de Bioética, 12, 1. https://doi.org/10.14422/rib.i12.y2020.001

Thiele, t. et al. (2017). Experience of disadvantage: The influence of identity on engagement in working class students' educational trajectories to an elite university. British Educational Research Journal Vol. 43(1), 49-67. https://doi.org/10.1002/berj.3251

Triana, C. P. C., y Parra, J. J. T. (2020). Estudio cualitativo del aprendizaje experiencial para equipos de trabajo organizacional. Revista de ciencias sociales, 26(3), 71-82. https://doi.org/10.31876/rcs.v26i3.33232

Trillo, F., Parada, A. y Bernárdez-Gómez, A. (2020). El impacto formativo de la enseñanza en la universidad: una experiencia de autoevaluación docente. Braz. J. of Develop., 6(9), p. 67817-67829. https://doi.org/10.34117/bjdv6n9-279

Valladolid, M. N., y Chávez, L. M. N. (2020). El enfoque cualitativo en la investigación jurídica, proyecto de investigación cualitativa y seminario de tesis. Vox Juris, 38(2), 69-90. https://doi.org/10.24265/voxjuris.2020.v38n2.05 